\title{
Multislice Computed Tomography Imaging of Diverticultis Complication: Colovenous Fistula
}

\author{
Maija Radzina, Andris Laganovskis, Mara Tirane, Ligita Zvaigzne, \\ Peteris Prieditis, Voldemars Bruns, Svetlana Lugovska \\ Pauls Stradins Clinical University Hospital, Riga, Latvia
}

\section{SUMMARY}

Acute colon pathology as diverticulitis is a frequent and important gastrointestinal disease, but the clinical diagnosis is often difficult to state. Several radiological studies have been used to assist in the diagnosis of acute diverticulitis (e.g. barium enema, ultrasound, and computed tomography (CT). Colovenous fistula is a rare complication of diverticulitis. We have analyzed MSCT imaging role in evaluation of such complicated form of diverticular disease.

Key words: multislice computed tomography, diverticulitis, colovenous fistula, hepatic portovenous gas.

\section{AIM OF THE DEMONSTRATION}

Europe is characterized by a high prevalence of the diverticular disease, which increases with age (prevalence of 8-12 cases per one million) (1). Lower gastrointestinal bleeding (occurring in about 5-15\% of patients with colonic diverticula) and infection, resulting in abcesses, peritonitis and perforation (yearly incidence $-4 / 100000$, occurring in about 15-20\%), are the most frequent complications (1). Colovenous fistula is a rare complication of diverticulitis and only few case reports exist in literature. Several radiological studies have been used to assist in the diagnosis of acute diverticulitis (e.g. barium enema, ultrasound, and computed tomography (CT). We have analyzed MSCT imaging role in evaluation of such complicated forms of diverticular disease.
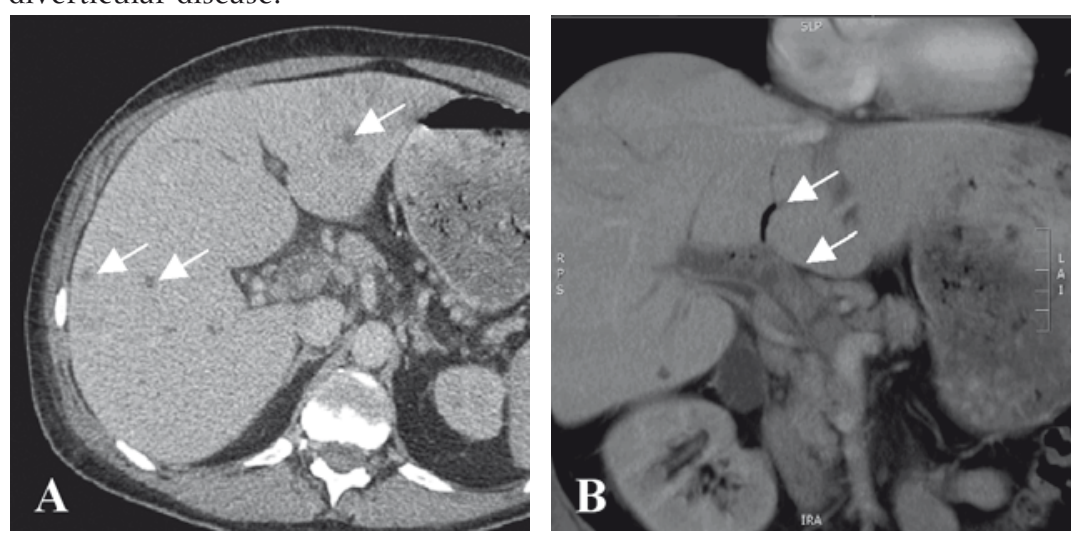

Fig. 1. A-CT, axial plane, post-contrast series - multiple, hypodense irregular lesions in the liver parenchymaseptic hepatic abscesses; B - CT reconstructions, coronal plane, post-contrast series - hepatic portovenous gas and inhomogenous, hypodense mass in the lumen of portal vein - thrombosis; C - CT, axial plane, postcontrast series - thickened wall and diverticula of sigmoid colon, pelvic free fluid - confirming diagnosis diverticulitis

There were also multiple left side colon diverticula, as well as irregular and thickened sigmoid colon wall, suggesting diagnosis of diverticulitis (Fig.1C).

\section{CASE REPORT}

Here we describe a case of an 46-year old male patient, that was admitted to emergency room with progressive epigastric and left lower quadrant abdominal pain during last month, followed by diarrhea, weakness, fever for 7 days and progressive jaundice at admission. Due to recent chronic hip pain a regular NSAIDs treatment was received. Clinical findings revealed severe septic status with liver function impairment and possible diagnoses of toxic hepatitis or cholangitis.

MSCT of abdomen with oral and intravenous contrast media revealed multiple parenchymal lesions in the enlarged liver, suggestive of septic abscesses (Fig 1A). Portal venous gas and thrombotic changes of portal system were detected on CT post-contrast scan (Fig. 1B). 


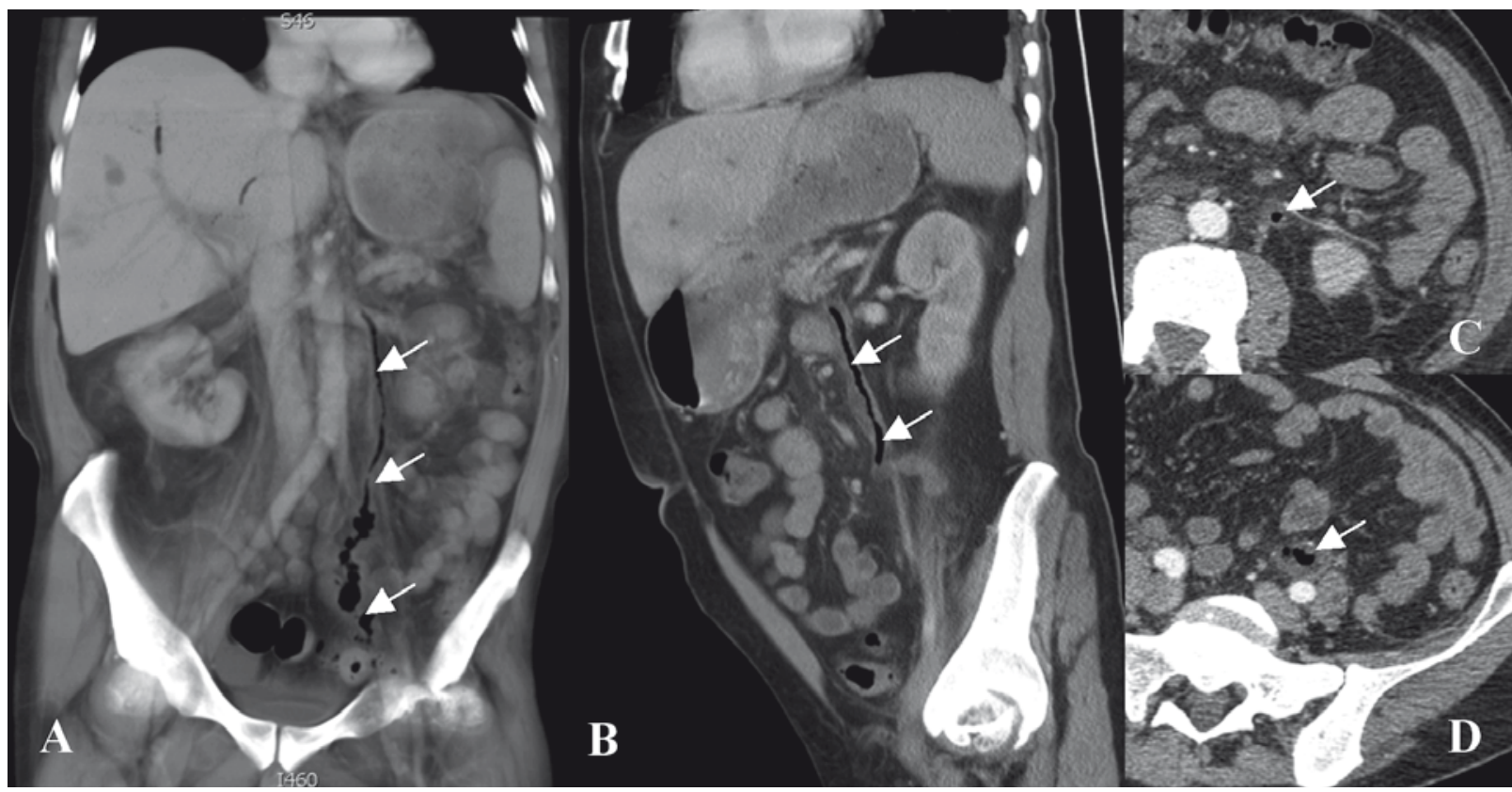

Fig. 2. CT reconstructions (A - coronal, B - sagittal, C, D - axial plane, post-contrast series show pathologic air-filled channel with well defined wall, extending from sigmoid colon up to epigastric region. Arterial branches along it's course and anatomical location suggest that inferior mesenteric vein is involved in colovenous fistula

Extensive peritoneal free fluid was noted (Fig.1C). A diagnosis of colovenous fistula (sigmoid colon to mesenteric vein) was proposed based on imaging findings and anatomy (Fig.3).

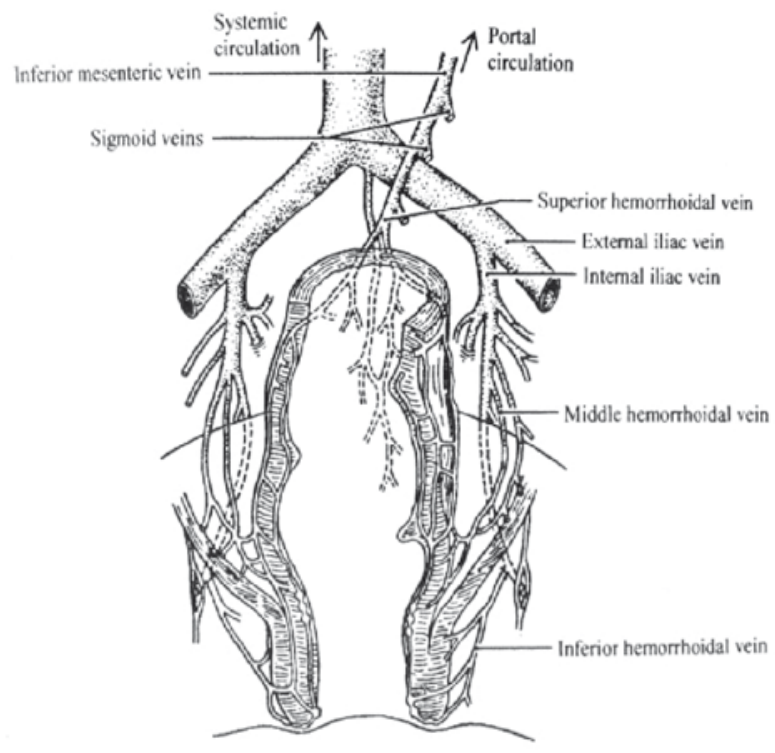

Fig.3. Anatomy of colon and hemorrhoidal veins (2)

Patient had surgery, but postoperative course was complicated with multiple laparatomies, drainage of liver and peritoneal abscesses. Follow-up CT demonstrated resolution of the portal vein gas and liver abscesses with persistent thrombotic changes in portal vein.

Cytology analysis revealed Enterococcus and E.coli presence in liver parenhchyma, peritoneal space and blood samples - confirming the diagnosis of colovenous fistula and sepsis.

After massive antibiotic treatment in ICU, during 6 weeks, eventually patient was discharged on $8^{\text {th }}$ week with improvement and closure of the colovenous fistula.

\section{DISCUSSION}

Complication of diverticulitis includes abscess, phlegmona, and peritonitis in different stages of disease. Fistula and sinus tract formation may involve blood vessels, bladder, vagina, uterus, ureter, and adjacent large and small bowel or abdominal wall, which may be visible by CT. (3)

True enterovenous and colovenous fistulas are rare but potentially lethal entities. The most common reported causes of fistulas involving the mesenteric small bowel and the colon are Crohn disease and diverticulitis. Inflammatory involvement of the inferior mesenteric vein complicating diverticulitis of the sigmoid colon can result in septic thrombophlebitis and the presence of gas within the vein. Portomesenteric vein gas is a rare complication of colonic diverticulitis, but the combination of the two conditions can lead to hepatic abscess (4) as it was seen in this case.

Differential diagnosis of hepatic portovenous gas (HPVG) is bowel ischemia or infarction, which is the most 
common cause of HPVG in adults and carries a mortality rate of $90 \%$ (5). Many other, nonischemic causes have been reported, such as intra-abdominal abscesses, bowel obstruction and distention, perforated gastric ulcer, gastric dilatation, gastrointestinal ileus, (acute hemorrhagic) pancreatitis, and ingestion of toxins. Less frequent are colon cancer, blunt abdominal trauma and hyperbaric decompression. Iatrogenic causes of HPVG can be barium enema, colonoscopy in patients with an inflammatory bowel disease or diverticulitis, endoscopic retrograde cholecystopancreatography with or without endoscopic sphincterectomy, and liver transplant (6). Colovenous fistulas are often detected unexpectedly on barium studies by observing intravasation of the contrast agent. Substantial barium intravasation reportedly carries a high mortality rate (3). We haven't used the barium enema imaging in this case, because a number of studies over the past decade have shown CT to be the preferable initial examination, due to its ability to demonstrate not only the extent of intramural inflammation but also the degree of pericolic disease, including intraperitoneal inflammation, perforation, and abscess formation (7). In this case the fistula could be missed on axial scans, interpreting it as part of normal colon.

Additional benefits of MSCT imaging include detailed multiplanar peritoneal cavity examination, CT angiography of portomesenteric vein system (e.g. thrombus, HPVG) and parenchymal lesion detection.

As surgical treatment is probably essential for this condition, the possibility of colovenous fistula should be borne in mind in patients with HPVG, especially if they have bacteraemia.

In conclusion - septic complications with development of colovenous fistula formation in diverticulitis are rare. MSCT imaging made notable impact in diagnosis and subsequent treatment of this patient. CT, by virtue of its superior contrast resolution and anatomic detail, allows more complete assessment of diverticulitis complications, including fistula and it's possible causes and outcome with use of postprocessing multiplanar reconstructions, as shown in this case.

\section{REFERENCES}

1. Delvaux M. Diverticular disease of the colon in Europe: epidemiology, impact on citizen health and prevention // Aliment Pharmacol Ther 2003; 18 (Suppl. 3): 71-74

2. Takahashi M, Fukuda K, Ohkubo U, Tokuhiro N, Tsuchiya R, Yoshie M, Hirai Y. Nonfatal Barium Intravasation into the Portal Venous system during Barium Enema examination // Internal Medicine 2004; 43:1145 - 1150

3. Lawrimore $\mathrm{T}$, Rhea JT.Computed Tomography Evaluation of Diverticulitis // J Intensive Care Med, 2006, 19(4): $194-204$

4. Sebastià C, Quiroga S, Espin E, Boyé R, AlvarezCastells A, Armengol M. Portomesenteric Vein Gas: PathologicMechanisms, CTFindings, andPrognosis // RadioGraphics 2000; 20:1213 - 1224

5. Pickhardt PJ, Bhalla S, Balfe DM. Acquired Gastrointestinal Fistulas: Classification, Etiologies, and Imaging Evaluation // Radiology 2002; 224: $9-23$

6. Heye S, Ghijselings L, van Campenhoudt M. Hepatic portal venous gas as a complication of diverticulitis with colovenous fistula: a case report // Emergency Radiology, 2002; 9:234 - 236

7. Strålin K, Lindgren R, Birgersson C, Vikerfors T. Gas within the liver and polymicrobial bacteraemia due to colovenous fistula: two cases in one month // Scand J Infect Dis. 2006; 38(1):66 - 8

\author{
Address: \\ Maija Radzina \\ Institute of Diagnostic Radiology, \\ Pauls Stradins Clinical University Hospital \\ Pilsonu str. 13, Riga, LV-1002, Latvia \\ E-mail; maija@mailbox.riga.lv
}

\section{Conflict of interest: None}

